Journal of Advanced College of Engineering and Management, Vol. 4, 2018

\title{
STRUCTURALLY SIMILAR SETS OF TRANSCENDENTAL ENTIRE FUNCTION
}

\author{
Bishnu Hari Subedi \\ Central Department of Mathematics \\ Institute of Science and Technology, Tribhuvan University, Kathmandu, Nepal \\ Email Address: subedi.abs@gmail.com, subedibbh@cdmathtu.edu.np
}

\begin{abstract}
In complex dynamics, the complex plane $\mathbb{C}$ is partitioned into invariant subsets. In classical sense, these subsets are of course Fatou set and Julia set. The latest results are concentrated on the partition of complex plane by the sets of the form I(f), K( f) and $B U(f)$. For a transcendental entire function $f$, the set $I(f)=\left\{z \in \mathbb{C}: f^{n}(z) \rightarrow \infty\right.$ as $\left.n \rightarrow \infty\right\}$ is an escaping set, the set $K(f)=\left\{z \in \mathbb{C}: \exists \quad R>0\right.$ such that $\left|f^{n}(z)\right| \leq R$ for all $\left.n \geq 0\right\}$ is a bounded orbit set and $B U(f)=\mathbb{C} \backslash(I(f) \cup K(f))$ is a neither escaping nor bounded orbit set. We study here basic similarity and topological similarity among the sets I(f), K(f) and BU(f). In our general review, we find both types of similarity occur among these sets and so we call these sets by structurally similar sets
\end{abstract}

Key Words: Escaping set, bounded orbit set, neither bounded nor escaping orbit set, structurally similar sets etc.

\section{Introduction}

We denote the complex plane by $\mathbb{C}$ and set of integers greater than zero by $\mathbb{N}$. We assume the function $\mathrm{f}: \mathbb{C} \rightarrow \mathbb{C}$ is transcendental entire function (TEF) unless otherwise stated. For any $n \in \mathbb{N}, \mathrm{f}^{\mathrm{n}}$ always denotes the nth iteratesof $\mathrm{f}$. A family $\mathcal{F}=\left\{\mathrm{f}\right.$ : $\mathrm{f}$ is meromorphic on some domain $\mathrm{X}$ of $\left.\mathbb{C}_{\infty}\right\}$ forms normal family if every sequence $\left(f_{i}\right)_{i \in \mathbb{N}}$ of functions contains a subsequence which converges uniformly to a finite limit or converges to $\infty$ on every compact subset $\mathrm{D}$ of $\mathrm{X}$. The Fatou set of $\mathrm{f}$ denoted by $F(f)$ is the set of point's $z \in \mathbb{C}$ such that sequence $\left(f^{n}\right)_{n \in \mathbb{N}}$ forms a normal family in some neighborhood of $z$ in the sense of Montel. A maximally connected subset of the Fatou set $F(f)$ is called Fatou component. The complement of Fatou set is called Julia set and it is denoted by J(f). Classically, it is known that the sets $F(f)$ and $J(f)$ form a fundamental partition of complex plane $\mathbb{C}$. The basic properties and structures of these sets can be found in the work of Bergweiler (1993), Carleson and Gamelin (1992), Hua and Yang (1998), Milner (2006) and Morosawa et. al (1999).

Based on the study of the basic properties and structures of Fatou set and Julia set, we find that these two sets are totally different. For example, Fatou set is largest open set, Julia set is smallest closed set, Fatou set is stable and Julia set is chaotic etc. Our concerns of study concentrate on the alternative dynamical partition of the complex plane by structurally similar sets depending on the nature of the orbits.

For any $z \in \mathbb{C}$, the sequence $\left(f^{n}(z)\right)_{n \geq 0}$ of iterates of function $f$ is called the orbit of $z$ under $f$. The orbit of any point in the complex plane may escape to infinity, it may be bounded, or it neither may escape nor bounded. In this paper, we are concerned with sets associated to above mention all three types of orbits.

The set of points whose orbits are escaped is called escaping set and actually, it is a set defined as follows.

Definition 1.1 (Escaping set). For a TEF f, the set of the form

$$
\mathrm{I}(\mathrm{f})=\left\{\mathrm{z} \in \mathbb{C}: \mathrm{f}^{\mathrm{n}}(\mathrm{z}) \rightarrow \infty \text { as } \mathrm{n} \rightarrow \infty\right\}
$$

Is called escaping set. Any point $\mathrm{z} \in \mathrm{I}(\mathrm{f})$ is called escaping point. 
For a TEF f, the escaping set I(f) was first studied by A. Eremenko (1989) together with formulation of the following two conjecture.

Conjecture 1.1: Each component of I( $f$ ) is unbounded.

Eremenko [6] himself proved that $\mathrm{I}(\mathrm{f}) \neq \varnothing, \mathrm{I}(\mathrm{f}) \cap \mathrm{J}(\mathrm{f}) \neq \varnothing, \mathrm{J}(\mathrm{f})=\partial \mathrm{I}(\mathrm{f})$. He also proved that all components of $\overline{\mathrm{I}(\mathrm{f})}$ are unbounded and conjectured that same may be hold for all components of $\mathrm{I}(\mathrm{f})$ which is the conjecture 1.1. The conjecture 1.1 has inspired many mathematicians and they have made substantial amount of work in transcendental dynamics. With remarkable attempts and efforts, much progress has been made in special cases and there are various partial results, but in general, the conjecture is still unsolved.

The set of points whose orbits are bounded is called bounded orbit set which is defined as follows.

Definition 1.2 (Bounded orbit set). For TEF f, the set of the form

$$
\mathrm{K}(\mathrm{f})=\left\{\mathrm{z} \in \mathbb{C}: \exists \mathrm{R}>0 \text { such that }\left|\mathrm{f}^{\mathrm{n}}(\mathrm{z})\right| \leq \mathrm{R} \text { for all } \mathrm{n} \geq 0\right\}
$$

is called bounded orbit set.

If $\mathrm{f}$ is a non-linear polynomial, the set $\mathrm{I}(\mathrm{f})$ is the basin of attraction of the point at infinity and hence a unbounded component of Fatou set and $\mathrm{K}(\mathrm{f})$ is known as filled in Julia set, a much studied compact bounded component of Fatou set. For transcendental entire function $f$, the set $I(f)$ was extensively studied by Eremenko (1989), Rippon and Stallard (2009, 2011, 2015), Rottesfusser and Schleicher (2005), and Schleicher and Zimmer(2003) and the set K( f) has received less attention and was studied by Bergweiler (2012) and Osborne (2013).

The set of points whose orbits are neither escaped nor boundedis called neither escaping nor bounded orbit set which is a set of the following form.

Definition 1.3 (Neither escaping nor bounded orbit set). For TEF f, the set of the form

$$
\mathrm{BU}(\mathrm{f})=\mathbb{C} \backslash(\mathrm{I}(\mathrm{f}) \cup \mathrm{K}(\mathrm{f}))
$$

is called neither escaping nor bounded orbit set.

For a non-constant polynomial function $\mathrm{f}$, we have $\mathrm{BU}(\mathrm{f})=\emptyset$. However for TEFf, $\mathrm{BU}(\mathrm{f}) \neq \varnothing$. This set was studied first time by Osborne and Sixsmith [12].

Note that the set $\mathrm{BU}(\mathrm{f})$ together with $\mathrm{I}(\mathrm{f})$ form a set $\mathrm{I}^{+}(\mathrm{z})$ of points whose orbits are unbounded and it is a set compliment to $\mathrm{K}(\mathrm{f})$ in $\mathbb{C}$. This type of set was studied first time intensively by Osborne, Rippon and Stallard (2015).

The structure of this paper is as follows. In section 2, we collect basic properties of the sets I(f), $K$ ( $f$ ) and $\mathrm{BU}(\mathrm{f})$ and on the basis of these properties, we find that there is a fundamental basic similarity among these sets. In section 3, we review topological properties mainly the connectedness and boundedness properties of these sets. Based on these properties, we find that there is also topological similarity among these sets. Finally, we conclude that both types of similarity occur among the sets I( $\mathrm{f}), \mathrm{K}(\mathrm{f})$ and $\mathrm{BU}(\mathrm{f})$, and we call these sets by structurally similar sets.

\section{Basic Similarity Among the Sets $I(f), K(f)$ and $B U(f)$}

In complex dynamics, the complex plane is partitioned by invariant subsets. The most important classical partition is by structurally different Fatou set and Julia set. We have here another partition by the sets $I(f), K(f)$ and $B U(f)$. We expect that these sets are structurally similar. The question arisesare the sets $I(f), K(f)$ and $B U(f)$ invariant? To look answer, we need the notion of invariant set. 
Definition 2.1(Completely invariant set). A set $U \subset \mathbb{C}$ is called forward invariant if $z \in U$ implies that $\mathrm{f}(\mathrm{z}) \in \mathrm{U}$. It is called backward invariant if $\mathrm{f}^{-1}(\mathrm{U})=\{\mathrm{z} \in \mathbb{C}: \mathrm{f}(\mathrm{z})=\mathrm{w} \in \mathrm{U}\} \subset \mathrm{U}$. It is called completely invariant if it is both forward and backward invariant.

The following simple result due to Osborne and Sixsmith [12] will be worth mentioned.

Theorem 2.1. For a TEF $\mathrm{f}$, each of the sets $\mathrm{I}(\mathrm{f}), \mathrm{K}$ ( $\mathrm{f}$ ) and $\mathrm{BU}$ ( f ) is completely invariant and together they form a dynamical partition of complex plane $\mathbb{C}$.

By this theorem 2.1, we can say that the complete study of dynamical plane $\mathbb{C}$ is possible via the sets $\mathrm{I}(\mathrm{f}), \mathrm{K}(\mathrm{f})$ and $\mathrm{BU}(\mathrm{f})$. We call a property of sets given by this theorem by first kind of basic similarity.

The following second types of basic similarities hold among the sets $I(f), K(f)$ and BU( $f)$.

Theorem 2.2. Let $\mathrm{f}$ be a TEF. Then

a. $\quad \mathrm{I}(\mathrm{f}) \neq \varnothing, \mathrm{K}(\mathrm{f}) \neq \varnothing$ and $\mathrm{BU}(\mathrm{f}) \neq \varnothing$

b. $\quad \mathrm{I}(\mathrm{f}) \cap \mathrm{J}(\mathrm{f}) \neq \varnothing, \mathrm{K}(\mathrm{f}) \cap \mathrm{J}(\mathrm{f}) \neq \varnothing$ and $\mathrm{BU}(\mathrm{f}) \cap \mathrm{J}(\mathrm{f}) \neq \varnothing$

c. $\quad J(f)=\partial \mathrm{I}(\mathrm{f}), \mathrm{J}(\mathrm{f})=\partial \mathrm{K}(\mathrm{f})$ and $\mathrm{J}(\mathrm{f})=\partial \mathrm{BU}(\mathrm{f})$.

The statements $\mathrm{I}(\mathrm{f}) \neq \varnothing, \mathrm{I}(\mathrm{f}) \cap \mathrm{J}(\mathrm{f}) \neq \varnothing$ and $\mathrm{J}(\mathrm{f})=\partial \mathrm{I}(\mathrm{f})$ were proved by Eremenko (1989)[Theorems $1,2], \mathrm{K}(\mathrm{f}) \neq \varnothing, \mathrm{J}(\mathrm{f})=\partial \mathrm{K}(\mathrm{f})$ and $\mathrm{K}(\mathrm{f}) \cap \mathrm{J}(\mathrm{f}) \neq \varnothing$ were stated by Bergweiler (2012) and $\mathrm{BU}(\mathrm{f}) \neq \varnothing$ and $\mathrm{BU}(\mathrm{f}) \cap \mathrm{J}(\mathrm{f}) \neq \varnothing$ were proved by Baker and Dominguez (2000) [Lemma 1], J(f) $=\partial \mathrm{BU}(\mathrm{f})$ was proved by Osborne and Sixsmith (2015) [ Theorem 1.1 (b)].

By this theorem 2.2, we can say that there is strong connection between these sets and the Julia set $J(f)$ as all these sets are non-empty like Julia set and all these sets intersect Julia set as well as boundary of all these sets is the Julia set. We call properties of the sets given by this theorem by second kind of basic similarity.

The following third type of basic similarity hold among the sets $I(f), K(f)$ and BU(f) .

Theorem 2.3. Let fbe a TEF. Then

a. all of these three sets I( f ), K( f) and BU( f)are neither open nor closed,

b. let $\mathrm{U}$ is a Fatou component such that $\mathrm{U} \cap \mathrm{I}(\mathrm{f}) \neq \varnothing, \mathrm{U} \cap \mathrm{K}(\mathrm{f}) \neq \varnothing$ and $\mathrm{U} \cap \mathrm{BU}(\mathrm{f}) \neq \varnothing$. Then $\mathrm{U} \subset \mathrm{I}(\mathrm{f}), \mathrm{U \subset K}(\mathrm{f})$ and $\mathrm{U \subset BU}(\mathrm{f})$.

Proof. (a). If $I(f)$ is open then $I(f) \subset F(f)$ which is a contradiction for theorem 2.2 (b). If $I(f)$ is closed, then $\mathrm{J}(\mathrm{f}) \subset \mathrm{I}(\mathrm{f})$ which is a contradiction for theorem $2.2(\mathrm{c})$. $\mathrm{K}(\mathrm{f})$ and $\mathrm{BU}(\mathrm{f})$ follows from the similar argument.

(b). If $U \cap B U(f) \neq \varnothing$, then $U \subset B U(f)$ for a Fatou component $U$, follows from[12, Theorem 1.1(a)]). The rest part follows from the similar argument.

The theorem 2.3 tells that each of the sets $\mathrm{I}(\mathrm{f}), \mathrm{K}(\mathrm{f})$ and BU( $\mathrm{f}$ ) neither open nor closed and if a Fatou component $U$ intersects each of these set, then by normality $U$ contained in each of them. Equivalently, every component of Julia set intersects each of sets $I(f), K(f)$ and BU(f). We call properties of the sets given by this theorem 2.3 by the third kind of basic similarity.

Definition 2.2.Two or more subsets of a complex plane are said to be basic similar sets if between or among them there exist first, second and third kind of basic similarity. Otherwise, the sets are said to be a similar. 
It is obvious that the sets $\mathrm{I}(\mathrm{f}), \mathrm{K}(\mathrm{f})$ and $\mathrm{BU}(\mathrm{f})$ are basic similar sets. Also the sets $\mathrm{K}(\mathrm{f})$ and $\mathrm{I}^{+}(\mathrm{z})$ are basic similar sets. On the other hand, the Fatou set $F(f)$ and Julia set $J(f)$ are a similar sets because between $F(f)$ and $J(f)$ there is only first kind of similarity.

Open Problem: Are there any other basic similar sets other than I(f), K(f) and BU(f)or K(f) and $\mathrm{I}^{+}(\mathrm{z})$ ?

\section{Topological similarity among the sets $I(f), K(f)$ and $B U(f)$}

In this section, we study connectedness and boundedness properties of the sets I(f ), K( $f$ ). On the basis of this study, we see that there is certain amount of similarity among these sets. If there is similarity, we call it by topological similarity, and the sets are said to be topologically similar.

Rippon and Stallard [Theorem 1.2] have provided conditions for the connectedness of the escaping set I(f). The parallel results were proved by Osborne, Rippon, and Stallard (2011)[Theorems 1.3, 3.1, 4.1,

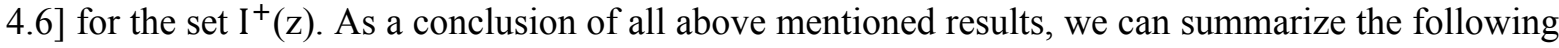
result regarding the connectedness of the set $\mathrm{I}(\mathrm{f})$.

Theorem 3.1: Let $\mathrm{f}$ be TEF. The escaping set I( $\mathrm{f}$ ) is either connected or it has uncountable many components, infinitely many of which are unbounded.

Osborne (2013)[Theorem 1.1] proved the following result concerning the connectedness property of the set $K(f)$.

Theorem 3.2: Let $\mathrm{f}$ be a TEF. Then $\mathrm{K}(\mathrm{f})$ is either connected or it has infinitely many components.

In the same paper, Osborne provided examples of TEFs for which $\mathrm{K}(\mathrm{f})$ is connected or totally disconnected. We refer examples 5.2, 5.3 and 5.4 for the transcendental entire functions that have totally disconnected $\mathrm{K}(\mathrm{f})$. For the function $\mathrm{f}(\mathrm{z})=\lambda \mathrm{e}^{\mathrm{z}}$ where $0<\lambda<\mathrm{e}^{-1}$, Fatou set $\mathrm{F}(\mathrm{f})$ contains the immediate basin of attracting fixed point. So that $K(f) \subset F(f)$ and since $F(f)$ is connected and $\overline{\mathrm{F}(\mathrm{f})}=\mathbb{C}$. It follows that $\mathrm{K}(\mathrm{f})$ is connected.

The following analogous result was obtained by Osborne and Sixsmith(2015) [Theorem 1.2(c)].

Theorem 3.3: Let $f$ be a TEF. Then either BU( $f)$ is connected or every neighborhood of any points in $\mathrm{J}(\mathrm{f})$ meets uncountable many components of $\mathrm{BU}(\mathrm{f})$.

The important fact we have to keep in mind here is that for particular TEF $\mathrm{f}$, the connectedness of $\mathrm{K}$ ( $f)$ may not imply the connectedness of $B U(f)$ and vice versa. For example, $K(f)$ is connected for the function $\mathrm{f}(\mathrm{z})=\lambda \mathrm{e}^{\mathrm{z}}$ where $0<\lambda<\mathrm{e}^{-1}$, while $\mathrm{BU}(\mathrm{f})$ is totally disconnected. Also, for particular TEF $f$, there may be both of the sets are connected or totally disconnected. For example, both sets K( f) and $\mathrm{BU}(\mathrm{f})$ are totally disconnected for the Fatou function $\mathrm{f}(\mathrm{z})=\mathrm{z}+1+\mathrm{e}^{\mathrm{z}}$.

The connectedness properties given in the theorems 3.1, 3.2 and 3.3 also hold for the set $\mathrm{I}^{+}(\mathrm{f})$. We refer [11] for more detailed study of this set.

Note that there are different conditions for connectedness of each of the sets $I(f), K(f)$ and $B U(f)$. See $[10,11,12,14$ and 17] for more detailed study of these sets. Our concern of study is not to analyze all these conditions but to see similarity on the connectedness of each of these sets.

On the basis of the results given in the theorems 3.1, 3.2 and 3.3, we call the similarity among the sets $\mathrm{I}(\mathrm{f}), \mathrm{K}$ ( f)and BU(f) by connectedness similarity or topological similarity. In other words, two or more subsets of the complex plane $\mathbb{C}$ are said to have connected similarity if these sets are either connected or they have infinitely many components. It is very obvious that the sets $I(f), K(f)$ and $\mathrm{BU}(\mathrm{f})$ or $\mathrm{I}^{+}(\mathrm{f})$ and $\mathrm{K}(\mathrm{f})$ have connectedness similarity. 
Baker and Dominguez (2000) [Theorem B] investigated that the Julia set J(f)is either connected or it has uncountable many components. Thus the sets $I(f), J(f)$ and $B U(f)$ also have connectedness similarity. Such type of connectedness does not hold for Fatou set, so it has no connectedness similarity with any of the sets $I(f), J(f), K(f)$ and $B U(f)$.

At the end of this section, we discuss the boundedness properties of sets $I(f), K(f)$ and $B U(f)$ in the sense that each of these set has unbounded components or not. It well known that every component of $\overline{\mathrm{I}(\mathrm{f})}$ is unbounded [6, Theorem 3]. Osborne and Sixsmith(2015) [Theorem 1.2(a)] have shown that iffhas no multiply connected Fatou components, then all components of $\overline{\mathrm{BU}(\mathrm{f})}$ are unbounded. It is known that the set K(f) (stated by Osborne ) is not bounded for TEF f. On the basis of these results, we can conclude the following results.

Theorem 3.4: Let $f$ be a TEF. Then the set $I(f)$ has at least one unbounded component Unfortunately, analogous result for the sets $\mathrm{K}(\mathrm{f})$ and $\mathrm{BU}(\mathrm{f})$ does not hold in general and so there is no boundedness similarity among the sets $\mathrm{I}(\mathrm{f}), \mathrm{J}(\mathrm{f})$ and $\mathrm{BU}(\mathrm{f})$.

We are going to define our basic term that we have already proposed.

Definition 3.1: Two or more subsets of the complex plane $\mathbb{C}$ are said to be structurally similar if between or among them there exist both basic and topological similarity.

It is obvious that the sets $\mathrm{I}(\mathrm{f}), \mathrm{K}(\mathrm{f})$ and $\mathrm{BU}(\mathrm{f})$ are both basic and topologically similar sets. Also the sets $\mathrm{K}(\mathrm{f})$ and $\mathrm{I}^{+}(\mathrm{f})$ are both basic and topologically similar sets. So these sets are structurally similar.

We have formulated the following conjecture as a conclusion of this paper.

Conjecture 3.1: There are no structurally similar sets other than $I(f), K(f)$ and $B U(f)$ or $K(f)$ and $\mathrm{I}^{+}(\mathrm{f})$ of TEF $\mathrm{f}$ in the complex plane $\mathbb{C}$. 


\section{References}

1. $\quad$ Baker, I.N and Dominguez, P. (2000). "Residual Julia sets”, J. Analysis 8, 121-137.

2. Baker, I.N and Dominguez, P. (2000). "Some connectedness properties of Julia set”, Complex Variables Theory Appl. 41, no. 4, 371-389.

3. Bergweiler, W. (1993). "Iteration of meromerphic functions". Bull. Amer. Math. Soc. (N.S.) 29 (2) 151-188.

4. Bergweiler, W. (2012). "On the set where iteration of entire functions is bounded", Proc.Amer. Math. Soc. 1403 (2012), 847- 853.

5. Carleson, L., and Gamelin, T.W. (1992). “Complex dynamics, Springer-Verlag”, New York.

6. Eremenko, A.(1989). "On the iteration of entire functions", Dynam. Sys. andErgod. Theory, Banach Center Publications, polish Scientific Publishers, 23, 339-345.

7. Hua, X.N., and Yang, C.C. (1998). "Dynamics of transcendental functions".(Asians Mathematical Series), Gorden and Breach Science Publishers, Hong Kong China.

8. Milner, J. (2006). "Dynamics in one complex variables (Third Edition)", Annals of Mathematical Studies, 160, Princeton University Press, Princeton, NJ.

9. Morosawa, S., Nishikura, Y., Taniguchi, M. and Ueda, T(1999). "Holomorphic dynamics", Cambridge Studies in Advanced Mathematics 66, Cambridge University Press.

10. Osborne, J.W. (2013). "Connectedness properties of the set where iterates of entire functions are bounded", Math.Proc.Cambridge Philos.Soc.155 (3),391-410.

11. Osborne J. W., and Rippon P.J., and Stallard, G.M. (2015). "Connected Properties of the set where the iteration of the entire functions are unbounded", arXiv: 1504.5611.v1 [math.DS].

12. Osborne, J. W. and Sixsmith D.J. (2015). "On the set where iterates of an entire functions are neither escaping nor bounded", arXiv: 1503.08077 v1 [math. DS]..

13. Rippon, P.J. and Stallard, G.M. (2009). "Escaping Point of entire functions of small growth", Math. Z., 261(3), 557-570.

14. Rippon, P.J., and Stallard, G.M. (2011). "Boundaries of escaping Fatou components”, Proc. Amer. Math. Soc. Vol.-139, No.-8, 2807-2820.

15. Rippon, P.J., and Stallard, G.M. (2012). "Fast escaping points of entire function", proc. London Math. Soc. 105, 787-820.

16. Rippon, P.J., and Stallard, G.M. (2013). "Baker conjecture and Eremenko's conjecture for functions with negative real zeros", J. Anal. Math, Res. Not., arXiv: 1208.3371v1.

17. Rippon, P.J., and Stallard, G.M. "Boundaries of univalent Baker domains", arXiv: 1411.6999 and to appear in Journal d'Analyse.

18. Rottesfusser, G. and Schleicher, D. (2008). "Escaping points of cosine family, In transcendental dynamics and complex analysis", Volume 348 of London Maths Society, Lecture Note Series", Page 396-424, Cambridge Uni. Press, Cambridge.

19. Schleicher, D., and Zimmer, J. (2003). "Escaping points of exponential maps", J. London Math. Soc. (2) 67, 380-400. 